\title{
TEMPO, INCERTEZZA E ISTITUZIONI. CONSEGUENZE DELL'INNOVAZIONE E RUOLO DELLA POLITICA
}

\author{
RAIMONDO CUBEDDU*
}

1. La mia relazione muove dalla riflessione che se per secoli, e per lo meno negli ultimi della vita dello stato moderno, abbiamo considerato la politica prevalentemente come una modalità di accelerazione di alcuni, o della generalità, dei processi sociali verso un fine che, in qualche o vario modo, era ritenuto possibile ed auspicabile, ora dobbiamo iniziare a riflettere sull'opportunità di continuare a farlo. Dobbiamo infatti chiederci se:

a) il fatto che il lasso di tempo che intercorre tra una qualsiasi scoperta scientifica e la sua diffusione sociale tende da decenni a restringersi sempre di più;

b) la circostanza che il tempo impiegato dalla politica a compiere delle scelte collettive è in costante e generale crescita;

c) la tendenza delle aspettative individuali a formarsi in contesti socio-culturali che finiscono per avere sempre minori relazioni con quelli in cui si esprimono politicamente; e,

d) il fatto che la grandi innovazioni e modificazioni riguardanti la vita individuale e sociale tendono a realizzarsi fuori dalla sfera pubblica, siano fenomeni che hanno trasformato e che muteranno ulteriormente tanto il tradizionale modo di intendere tanto la politica, quanto il modo e le forme istituzionali in cui si esprime.

Di conseguenza - sempre che tutte queste assunzioni siano vere- non possiamo evitare di chiederci quale saranno i futuri

* Università di Pisa. Socio corrispondente. 
ruoli della politica e dello stato, e se uno dei due potrà sopravvivere, ed eventualmente in quale forma, alla scomparsa dell'altro.

Da secoli, infatti, siamo così avvezzi a identificare la teoria politica con lo stato (negli ultimi decenni, per di più, molti studiosi hanno compiuto anche un altro passo, per così dire, 'in avanti', scambiandola con la costituzione, e lasciando aperta soltanto l'opzione se si tratti di quella nazionale o di qualchedun altra) che fatichiamo ad immaginare la possibilità della politica senza lo stato. A mio avviso si tratta di una possibilità con la quale è il caso di misurarci per il fatto che - per lo meno per quella parte della filosofia politica che non dimentica i classici (o che almeno non pensa che tutti i classici siano tedeschi) - mentre lo stato è una forma storica di organizzazione politica, per ora (e mi associo a quanti se ne dispiacciono), la possibilità che si possa fare a meno della politica non è stata dimostrata in maniera convincente.

Per introdurre il tema, che così espresso può apparire arduo -e che comunque è quello del ruolo, o della funzione, della politica nelle società contemporanee caratterizzate da un elevato indice di complessità- prenderò le mosse da due esempi, tratti della vita di ogni giorno e sotto gli occhi di tutti: la lavatrice e internet.

Senza che lo volesse chi l'ha inventata, la lavatrice ha mutato i rapporti familiari, e quindi la società, più dei teorici del 'diritto di famiglia'. Capisco che esposto in questi termini il mio ragionamento possa apparire alquanto bizzarro. Ma se si riflette un po' sull'esempio ci si può rendere conto immediatamente della relazione tra innovazione scientifico-tecnologica, mutazione delle aspettative individuali, cambiamento sociale, ruolo del diritto e della politica. Se si vuole, la questione può essere vista anche da una prospettiva meno legata alla contingenza: aumentando il tempo a disposizione di una delle componenti della società (la donna-casalinga) la tecnologia ha trasformato la società dando vita anche a nuovi valori e a nuovi modelli di comportamento.

Il caso di internet è ancora più interessante per il fatto che non soltanto, come la lavatrice, consente di ridurre il tempo necessario 
per svolgere attività tradizionali (anche se non dobbiamo dimenticare che ha dato vita ad altre attività 'impegnative' come, ad esempio, quella di 'passare tempo su internet'), ma ha decentrato e diffuso l'informazione e la conoscenza col risultato di renderle accessibili ad una numero sempre maggiore di individui e di rivoluzionarne le forme tradizionali di trasmissione (scuole, biblioteche, stampa, etc.). Ha mostrato, internet, che la tecnologia è in grado di risolvere problemi (come quello dell'educazione) che prima si riteneva potessero, e dovessero, avere soltanto una soluzione politica, ha accelerato ed allargato gli scambi riducendone il tempo e, con tutto ciò, ha contribuito a generare una moltitudine incontrollabile di aspettative, tra le quali, dal nostro punto di vista, spicca quella di attendersi dalla politica ciò che, e a prezzi molto contenuti, è già possibile avere nella o tramite la rete.

Queste due rivoluzioni, ed altre se ne potrebbero aggiungere e se ne aggiungeranno, anche se hanno delle evidenti implicazioni politiche, non sono però delle rivoluzioni politiche in senso tradizionale. Esse, infatti - e si tratta dell' aspetto decisivo della questione- avvengono fuori dal contesto decisionale della sfera pubblica e, forse, nessuno dei loro padri aveva previsto che potessero avere conseguenze tanto vaste; e neanche che potessero proporsi come soluzione di problemi che allora non si immaginavano, e che accanto a nuovi problemi avrebbero prodotto anche nuove ed altrettanto inaspettate opportunità. Ciò detto a proposito dell'imprevedibilità delle conseguenze delle innovazioni nel campo delle idee, e soprattutto nel campo scientifico-tecnologico (ciò che fa risultare sempre meno fondato quel poco che resta della distinzione tra 'scienze morali' e 'scienze naturali'), resta da dire che oggi nessuno stato potrebbe ormai combatterle se non al costo di un isolamento e di forme di repressione costose quanto inefficaci.

Tutto questo induce a chiederci quale sarà la funzione dello stato e della politica in un mondo in cui non potranno più avere la parola decisiva sulla nascita e neanche sulla diffusione delle idee. In un mondo nel quale il tempo in cui le idee - delle quali è impossibile controllare politicamente e socialmente i processi di nascita e di diffusione - tendono sempre più rapidamente a 
trasformarsi in conoscenza, e di qui in nuove opportunità ed aspettative, distribuite a loro volta in maniera casuale ed instabile, dando vita, altrettanto rapidamente, a fenomeni sociali che talora chiedono qualcosa alla politica, e che talaltra, e sempre più spesso, la fanno apparire superflua.

Da questo punto di vista, se internet è un 'bene pubblico' che non viene prodotto da nessuna istituzione pubblica, possiamo pensare (o sperare) che nel futuro il numero di tale tipologia di beni possa crescer a tal punto da rendere superflui, se non la politica, i suoi costosi ed inutili riti?

A quest'insieme di circostanze, di per se stesse nuove, bisogna aggiungere che a rendere le cose ancor più complicate, o forse, più schiettamente, a peggiorarle, è anche la circostanza che - per effetto (ma non solo) della frammentazione sociale prodotta dal modo in cui tale accelerazione ridistribuisce il 'tempo nella società' (fenomeno per certi versi analogo a quello della distribuzione casuale della conoscenza) - gli individui tendono a sviluppare nuove e non complementari aspettative. Di conseguenza, il numero dei cittadini che condividono i 'vincoli informali' tende a diminuire per il fatto che le scelte individuali che si generano in conseguenza di tale fenomeno possono essere anch'esse non complementari e dar vita a nuovi 'vincoli informali' i quali implicano nuove regole e nuovi 'vincoli formali' (il cosiddetto 'processo di cambiamento istituzionale').

Sembra quindi sia giunto il momento di abbandonare quel passato di ottimismo che sosteneva che il progresso della conoscenza, sia scientifica, sia morale, avrebbe risolto in maniera armonica i contrasti derivanti dalla scarsità di beni, o che $l^{\prime}$ incremento e la diffusione della conoscenza avrebbero ridotto i contrasti derivanti dalla loro scarsità, se non altro razionalizzandone la distribuzione. Erano gli anni in cui la politica si sentiva onnipotente e in cui da molti era ritenuta tale.

Come se non bastasse - ed persino questo sta avvenendo sempre più spesso-quando la politica riesce, in qualche modo, a 'regolare' e a distribuire le conseguenze positive e negative connesse alla diffusione sociale di tali novità (quel che in termini tecnici passa per 'allocazione dei diritti e delle opportunità', o per tentativi di realizzare la 'giustizia sociale'), la normativa 
appena varata viene superata da ulteriori novità le quali -il più delle volte- la rendono inutile e, nella prospettiva dello sviluppo, talora anche dannosa.

Di conseguenza, per quanto tali novità possano essere di tipi diversi, e, come si è visto, provenire da disparati ambiti della creatività umana, esse mettono comunque in luce due cose. La prima è che la politica sembra aver preso il carattere della progettualità e dell'imperio, e sembra ridursi ad elaborare criteri di distribuzione (che possono essere ispirati da ideali religiosi, etico-ugualitari, libertari, di utilità sociali, o altri ancora), ossia a regolare gli effetti, più o meno previsti e/o desiderati, di 'iniziative' altrui. La seconda è rappresentata dalla circostanza che il tempo in cui gli individui si aspettano (o pretendono) che vengano soddisfatte le loro pretese (da non confondere col tempo biologico che, al contrario, in questi decenni si è allungato) tende sempre meno a coincidere col tempo impiegato dalla politica a compiere delle scelte.

Non soltanto col tempo che le regole impiegano ad affiorare, affermarsi ed imporsi, ma anche col tempo che impiega la politica a produrre legislazione rispettando i requisiti di astrattezza, universalità ed imparzialità. Erano, è vero, i requisiti che la tradizione politico-costituzionale liberale aveva dovuto attribuire alla produzione legislativa del diritto per renderla in qualche modo accettabile. Tuttavia, per quanto si possa dire che ormai quei requisiti siano insoddisfacenti, certo non sono dannosi quanto quelli delle norme 'finalistiche' destinate ad essere superate dallo sviluppo della scienza. Tali norme, infatti, se non abrogate tempestivamente, finiscono per proteggere le oasi di privilegio che sopravvivono al cambiamento e che, quando non lo rallentano, lo rendono, per il semplice fatto della loro persistenza, ancora più incerto.

2. In via preliminare tutto questo induce a riflettere su una serie di circostanze.

La prima è che la conoscenza ed le aspettative tendono a sorgere, a svilupparsi e a diffondersi seguendo modalità di trasmissione delle informazioni (e, lo ripeto, fa poca differenza cercare di distinguerle secondo la vecchia distinzione tra 
conoscenza scientifico-tecnologica e conoscenza morale) che non sono più controllabili, o gestibili, dalla politica.

La seconda - e si tratta sempre dell'esempio di un'innovazione, questa volta nel campo della religione e delle pratiche individuali e sociali che vi si legano- è che la politica si mostra sempre più in affanno nel controllare le ripercussioni sociali, ed anche economiche, connesse sia alla pratica delle religioni e confessioni tradizionali dell'Occidente, sia all'inserimento nel suo contesto di valori e di regole sociali di individui e di gruppi appartenenti ad altre religioni.

La terza è forse giunto il momento di chiedersi se le modalità in cui avvengono le decisioni politiche - modalità che nei paesi democratici occidentali sono sostanzialmente quelle teorizzate dalla tradizione del costituzionalismo liberale e dalla teoria milliana del governo- siano ancora efficienti e valide in un mondo che da allora ha subito tanti e così radicali cambiamenti.

La quarta è che se fino a pochi decenni fa si pensava che la politica dovesse occuparsi della distribuzione dei beni e restare indifferente alla circolazione e alla diffusione delle idee e del tempo, ora, come si è visto, si avverte sempre più chiaramente che tale posizione non è più sostenibile per il fatto che la diffusione delle idee - l'economia della conoscenza- produce asimmetrie conoscitive che rendono sempre più tenui i 'vincoli informali' (o valori condivisi) all'interno di un" associazione politica'; modifica in continuazione le aspettative e i valori individuali e sociali; pone sempre nuove, onerose e contraddittorie domande alla politica.

La quinta è che l'uso dello strumento dell'obbligazione politica, ovvero degli strumenti coercitivi fondati su scelte collettive, tende a diventare sempre più difficile, gravoso e meno efficace per via del fatto che la conoscenza (e quindi lo sviluppo economico) tende a collocarsi dove ritiene (magari sbagliando o seguendo trends di breve periodo) di trovare l'ambiente migliore.

La sesta è che si accetta questa logica, la politica finisce per trasformarsi in una modalità di rallentamento del cambiamento (che può avere anche degli effetti salutari in quanto concede più tempo per capire e per adeguarsi ai cambiamenti), ma il cui costo è la dislocazione dell'innovazione in ambienti ad essa più 
favorevoli, e il cui risultato è di lasciare inalterate le aspettative individuali e sociali (per via della facilità di diffusione della conoscenza e di accesso alla medesima), e di ridurre drasticamente la quantità di risorse a disposizione della società e della politica.

In altre parole, gli stati che non riescono a seguire i ritmi del cambiamento vengono saltati dai suoi effetti positivi pur recependone quelli negativi: crescita esponenziale delle aspettative individuali e sociali non complementari e, sempre più spesso, pure prive di realismo.

Per cercare di uscire dall'impasse, non sembra tanto necessario buttare all'aria la tradizione costituzionale liberal-democratica, quanto rendersi conto che la politica non può controllare e dirigere il cambiamento imponendogli il proprio tempo. E non si può neanche pensare che la politica e i politici resisi conto della loro sostanziale inutilità decidano di dissolversi.

Appare semmai necessario che filosofi politici e politologi prendano atto che molto del loro armamentario normativo è diventato inadeguato, caduco, e inizino a chiedersi quali siano le reali conseguenze del fatto che se $i$ fenomeni sociali sono fenomeni culturali, e non naturali, non soltanto non tendono spontaneamente verso una sola direzione, ma, molto spesso, non tendono 'naturalmente' in nessuna direzione e, tanto meno, verso il meglio. Infine, se anche tendessero verso la progressiva realizzazione di una sorta di 'ottimo paretiano', tale realizzazione non avverrebbe nei tempi richiesti da una parte più o meno ampia delle componenti della società. Detto altrimenti, per via delle diverse capacità di percezione, non potrebbe avvenire simultaneamente per tutti.

Quello su cui intendo richiamare l'attenzione è, in definitiva, che la conoscenza e le aspettative individuali sono caratterizzate da una dimensione temporale che non corrisponde a quella delle istituzioni e, in particolare, a quello della politica. Inoltre, quest'ultima, in tali circostanze, potrebbe fare ben poco perché non è immaginabile che una forma decisionale che non può che avere una delimitazione geografica possa controllare fenomeni di nascita, diffusione, affermazione e cambiamento di conoscenze che si muovono invece in una dimensione globale.

Di conseguenza, se il fine delle istituzioni è quello di produrre certezza o indicazioni attendibili in merito alla possibilità di 
realizzazione (ossia ai costi e al tempo necessario) delle aspettative individuali e/o sociali, la politica si trova ora nella disagevole situazione di dover prendere atto della propria incapacità di produrre tale tipo di certezza.

All'incremento del bisogno sociale di certezza finisce così per corrispondere una crescente incapacità della politica di produrne nel tempo atteso dagli individui. Ma questa situazione genera, a sua volta, ulteriore incertezza e, paradossalmente, maggiore domanda di politica. E così, chi pensava di potersi liberare se non della politica per lo meno dello stato incontra una cocente delusione. Forse perché, avendo immaginato che fossero progettualità e accelerazione, si trova a dover prendere atto che erano, e sono, soltanto dei tentativi organizzati di ridurre l'incertezza.

3. Il ruolo che il tempo svolge nelle vicende umane è stato così a lungo trascurato nelle scienze sociali che anche nel linguaggio comune si dice che le buone regole producono buoni risultati; purtroppo senza specificare quando. Soltanto recentemente gli studiosi delle scienze sociali si sono resi conto del fatto che gli individui e i gruppi (più o meno organizzati) tendono sempre più a chiedere alla politica risposte ai propri bisogni soggettivi in tempi di cui, ovviamente, vogliono essere anche giudici.

In altre parole, non rassegnandosi più ai tempi della politica perché ritenuti a torto o a ragione troppo lunghi, questo tipo di individuo non si chiede più se le sue aspettative siano legittime, plausibili, o realistiche, se siano gerarchizzabili e compatibili con altre aspettative sociali, e neanche se sia esso stesso disposto a mantenerle nel tempo. Chiede alla politica di tutto, e rimane insoddisfatto se le sue richieste vengono dilazionate o eluse.

Alle origini di questo fenomeno è probabilmente la scomparsa della distinzione tra diritti individuali ed universalizzabili (che dovevano essere garantiti, assicurati o realizzati dalle istituzioni politiche) e aspettative individuali che, nella loro forma soggettiva, dovevano essere realizzate autonomamente dagli individui stessi all'interno del quadro rappresentato dal diritto (inteso come insieme di norme generali ed astratte) e dalla costituzione.

Per avere indicazioni su come ciò sia successo bisognerebbe rivolgere lo sguardo alla trasformazione (o metamorfosi) che i 
'diritti naturali' hanno subìto negli ultimi decenni e, forse, prendere anche in considerazione gli effetti della rottura di quel legame tra legge naturale e diritti naturali o umani, che ha lasciato questi ultimi privi di un qualche legame con la metafisica, con la teologia o anche con un qualche concetto di ragione. Riservandoci tale analisi per un'altra occasione, si può ora dire che una delle cause di quanto prima accennato può forse essere la dilatazione dei diritti (umani, sociali, alla salute, al lavoro, alla casa, al lavoro, ad una vita sessuale felice, etc.) che, facendo cadere la distinzione tra diritti naturali e aspettative, ha reso inutile e obsoleta quella distinzione tra sfera privata e sfera pubblica che, nel bene o nel male, costituiva da qualche secolo il cardine della tradizione politica occidentale.

Chiedendo allo stato di occuparsi di ogni problema e di realizzare ogni aspettativa, la distinzione pubblico/privato è così caduta e la politica, con esiti sempre meno soddisfacenti, si è trovata, anche contro la propria volontà, a doversi preoccupare di tutto e ad essere valutata non per la sua capacità di fornire a prezzi accettabili beni pubblici e certezza, ma per la sua capacità di soddisfare ogni tipo di soggettiva e transitoria aspettativa.

La politica, per quanto certe tradizioni ideologiche abbiano favorito tale cambiamento più di altre, si è comunque ritrovata impreparata di fronte a tante e nuove aspettative.

Ciò detto, non bisogna dimenticare che tale trasformazione e attenuazione della distinzione tra sfera pubblica e sfera privata, son state rese possibili, e in una certa misura anche prodotte - ancora una volta come risultato inaspettato o indesideratodallo sviluppo delle informazioni e della conoscenza disponibili, il quale ha fatto sì (o a indotto a credere) che in taluni casi la 'risposta politica' a certi problemi non fosse più necessaria. Sia per il fatto che essi potevano essere risolti anche da un mercato concorrenziale $\mathrm{o}$, più semplicemente, dallo sviluppo della conoscenza scientifica (basti pensare al ridimensionamento dello stato nella produzione di educazione in seguito ad internet); sia perché tutto ciò ha reso difficile dare una risposta circa la natura privata o pubblica delle conseguenze di certe scoperte o invenzioni: si pensi, ad esempio, alla difficoltà di collocare in una delle due sfere molte delle possibili applicazioni 
sociali delle scoperte nel campo delle tecniche di produzione della vita.

Come soluzione a questa serie di problemi si possono immaginare le seguenti opzioni:

a) ristabilire una distinzione tra diritti individuali (universalizzabili) e aspettative (soggettive) fondando i diritti su una sorta di legge naturale rivisitata. Soluzione difficile dal punto di vista filosofico e comunque realizzabile in tempi imprevedibilmente lunghi e con l'uso di dosi più o meno massicce di coercizione, o di 'educazione', per attuare una nuova e migliore allocazione di diritti.

b) diminuire, tramite una serie di riforme istituzionali, il tempo della decisone politica. Anche in questo caso valgono però, in buona parte, le osservazioni alla soluzione precedente per via del fatto che la creazione di 'vincoli informali' non è facilmente, né in tempi brevi, conseguibile tramite l'uso di strumenti politici o educativi.

La seconda soluzione è a mio avviso quella più praticabile. Soprattutto se si adotta un approccio che tragga ispirazione dalla teoria delle scienze sociali 'austriaca' e da quella 'neoistituzionalistica', e se si tiene conto del fatto che il recente volume di Douglass C. North, Understanding the Process of Economic Change, del 2005, apre un consistente spiraglio in tale direzione anche se non chiarisce del tutto come sia possibile modificare i vincoli informali tramite quelli formali in un ambiente in cui questi ultimi si possono sviluppare senza subire condizionamenti da parte dei primi. Come può avvenire nel caso di aspettative che si modificano in un ambiente legale sulla base di informazioni e di conoscenze che provengono dall'esterno e che non possono essere filtrate e selezionate dal potere politico.

Tutto ciò implica comunque una ridiscussione delle teorie $\mathrm{e}$ delle finalità della politica e richiede una riflessione sulla natura e sulla funzione dello stato moderno - il modello sul quale si è costruito il nostro concetto di politica e di costituzione- che prenda atto del fatto che per quante critiche possano essere rivolte allo stato, non si è ancora pensato a come e con che cosa sostituirlo. 
Non si tratta di buttare all'aria tutto, ma, più realisticamente, di sottoporre al vaglio le soluzioni proposte dalle tradizioni della filosofia politica che maggiormente hanno resistito alla critica corrosiva dei tempi, e di migliorarle aggiornandole sia alle nuove realtà, sia alle nuove aspettative.

Non è allora una caso che il più delle volte, quando si parla di un sistema istituzionale e delle scelte che vi avvengono, si dia per scontato che gli individui abbiano a disposizione il medesimo tempo e che le loro scelte siano motivate dal riferimento a valori $\mathrm{o}$ ad aspettative più o meno razionali e, più comunemente, più o meno connesse alle conoscenze di cui dispongono. Molto meno frequentemente tali valori, aspettative e conoscenze vengono messe in relazione all'aspettativa soggettiva di tempo individuale. Il fatto che quest' ultima sia difficilmente definibile o quantificabile, fa sì che nella formulazione di una teoria delle scelte le aspettative soggettive di tempo siano, sovente, tacitamente trascurate.

Ciò nonostante, non essendo la conoscenza 'data', le teorie esplicative dell'azione fondate sull'analisi della conoscenza individuale possedute dagli agenti, sono di grande rilevanza. Infatti, così come una società non è composta da individui dotati della medesima conoscenza, così non è costituita né da individui che hanno avuto a disposizione lo stesso tempo per acquisire conoscenze (e lasciamo da parte quali siano le loro doti naturali e la quantità e qualità di conoscenza acquisita), né da individui che hanno a disposizione lo stesso tempo per realizzare fini che hanno elaborato in relazione alla conoscenza posseduta.

$\mathrm{E}^{\prime}$ altresì risaputo che una società può essere definita 'buona' in relazione alla solidità ed estensione dei 'vincoli formali ed informali' che legano i suoi componenti, e che al suo interno deve esserci una distribuzione di conoscenza e di tempo tale da invogliare gli individui a tessere relazioni reciproche di scambio in assenza delle quali una società ristagnerebbe nella noia. Ed è anche noto, anche se più difficilmente quantificabile, che una società caratterizzata da marcate asimmetrie nella distribuzione di tempo, conoscenze e risorse sarebbe una società troppo conflittuale per poter essere definita 'buona'. Come pure che non potrebbe essere definita in tal modo una società in cui gli individui impiegano tempi molto diversi per raggiungere fini 
posti come uguali. Alle volte questa diversità viene attribuita alle dotazioni iniziali, altre alla conoscenza individualmente posseduta. E tuttavia, anche presupponendo che gli individui abbiano a disposizione la stessa 'quantità di tempo', queste diversità iniziali non si annullerebbero neanche in un periodo più o meno lungo (mentre sappiamo, per dirla con Keynes, che «nel lungo periodo .......»).

La rilevanza del fattore tempo è data appunto dal fatto che quanto prima detto non avviene, e che avverrebbe ancor meno se la dotazione individuale di conoscenza e la sua quantità astrattamente disponibile e dispersa nella società variassero nel tempo (in tal caso, infatti, produrrebbero ulteriore asimmetria nella sua distribuzione). Di conseguenza, per quanto la politica, soprattutto se si avvale di strumenti coercitivi (la cui efficacia è comunque limitata nel tempo), possa rendere possibili molte cose, non le è possibile 'distribuire tempo'. Tanto meno secondo criteri che tengano conto dell'utilità sociale, dei criteri di giustizia sociale e delle aspettative individuali. Pertanto, se si considera tale circostanza, una 'società buona' è impossibile, come è impossibile intenderla come un'entità collettiva che miri alla realizzazione di un 'bene comune'. Non è un caso che quanti l'hanno teorizzata hanno dovuto teorizzare la preminenza della conoscenza sociale su quella individuale, e hanno elaborato anche modalità per preservarla da fenomeni esogeni e criteri di distribuzione ispirati a valori etici sottratti alla disponibilità $o$ all'interpretazione degli individui. Il fatto che non si sia mai raggiunto l'obiettivo è, a mio avviso, da mettere in relazione al fatto che si sia da una parte trascurato l'aspetto della distribuzione individuale del tempo, e dall'altra parte al fatto che la conoscenza e l'eticità non si trasmettono geneticamente. Casi in cui l'obiettivo diverrebbe, sia pure in tempi imprevedibilmente lunghi se non connessi ad una trasmissione casuale dei geni, forse probabile. Se invece si volesse ridurre il tempo, bisognerebbe intervenire, sempre che sia possibile, sul meccanismo di trasmissione dei geni (eugenetica).

Venendo al dunque, tutte queste asimmetrie temporali, genetiche e gnoseologiche possono indurre parte degli individui a credere che la migliore soluzione dei loro problemi risieda in 
una certa gerarchia di priorità tra scelte collettive ed individuali ed altri - lasciando da parte i casi estremi di quanti li vorrebbero tutti risolti da scelte collettive e quanti soltanto da scelte individuali- in una gerarchia di priorità tra scelte individuali e collettive. Il che, pur essendo del tutto svincolato dalla reale possibilità di risolvere quei problemi tramite scelte collettive o individuali, finisce sia per far carico alla politica della realizzazione di aspettative che possono avere maggiori relazioni con la fantasia individuale di quante ne possono avere con le reali capacità e possibilità della politica, sia per determinare un regime di scelte che non hanno relazione con alcuna assiologia delle medesime ma piuttosto con le credenze diffuse. Poco male, se non fosse che una simile situazione determinerebbe anche la mancata, o non soddisfacente, realizzazione di quelle finalità le quali si potrebbero invece realizzare se non si fosse costretti da vincoli istituzionali magari 'stupidi' ma comunemente ritenuti migliori.

Di qui, per dirla in breve, l'importanza del 'fattore tempo'. Soprattutto in situazioni caratterizzate dal frequente e repentino mutare della conoscenza e delle modalità della sua produzione e distribuzione, che portano a scardinare le gerarchie sociali, di valori, le credenze religiose e sociali e, in tal modo finiscono per privare gli individui di punti di riferimento stabili sulla cui base orientare comportamenti in vista del conseguimento di fini e del calcolo del loro costo.

4. Si affaccia, in questo modo, un altro tema, quello della stupidità individuale e sociale, che la democrazia ha dovuto rimuovere o negare onde evitare di essere anch'essa definita come un regime stupido: vale a dire un regime che non consente di individuare la miglior soluzione ai problemi individuali, ma che fissa criteri di distinzione tra sfera pubblica e privata che dipendono da una singolare ed imprevedibile combinazione della distribuzione della conoscenza (e quindi della stupidità). Il problema è allora rappresentato dalla domanda se un regime democratico può consentire di fare una distinzione tra la conoscenza socialmente utile e quella che tale non può essere definita, e di identificare ed arginare gli effetti perversi della stupidità individuale e della sua connessione al fenomeno della 
mutazione e dell'incremento della conoscenza e delle aspettative. Nel senso che in una situazione statica la stupidità è più facilmente individuabile e controllabile di quanto non lo sia in una situazione caratterizzata dal frequente mutare della conoscenza che, rendendo tutti incerti li rende anche più stupidi.

Il discorso, in altre parole, potrebbe essere riassunto chiedendoci se la teoria democratica sia adeguata a tempi così complessi e caratterizzati sia dal frequente mutare della conoscenza, sia dalla diminuzione del tempo in cui avvengono le ricadute sociali di tale mutamento. In questo modo, tuttavia, oltre alla categoria dello 'stupido per natura', bisognerebbe elaborare anche la figura dello 'stupido per cultura'. Di un individuo che per motivi di attaccamento a convinzioni fondate (path dependence), al proprio stile di vita, non è disposto - perché lo ritiene troppo costoso o inutile in relazione al tempo che ritiene di avere a disposizionea cambiare la propria esistenza e le proprie aspettative in relazione alle trasformazioni prodotte dall'ingresso e dalla distribuzione di nuove conoscenze.

Di conseguenza, è diventato pressoché impossibile chiedersi quale sia ormai diventato il ruolo della politica e quali siano le sue reali capacità di risolvere situazioni caratterizzate dalla predominanza di 'stupidi per cultura'. Le scelte politiche, in definitiva, non possono essere affidate alla selezione dei componenti di organi decisionali fatta da un elettorato il quale, se non prevalentemente in condizione di 'stupidità', può bensì decidere per lo meno in condizione di ignoranza.

Se la libertà consiste nel diritto ad essere diversi occorre quindi chiedersi come si possano tollerare procedure decisionali che tendono ad uniformare le finalità e gli stessi processi decisionali pur sapendo che il loro esito sarà comunque insoddisfacente.

Anche la possibilità del Libertarianism, che pure è la tradizione della filosofia politica più lontana dalla tradizione democratica per il fatto che non prevede (o quasi) scelte collettive, risulta così connessa alla possibilità che quelle individuali si realizzino nei tempi ed ai costi previsti. Ma questo sarebbe possibile soltanto se tutti gli attori coinvolti fossero pienamente razionali, dotati di una conoscenza completa e non cambiassero le loro preferenze. 
Ciò che è impossibile. Un'altra possibilità, ma anch'essa improbabile data la sempre maggiore differenziazione nella distribuzione della conoscenza, è legata alla circostanza che le preferenze individuali siano naturalmente complementari e che tale complementarietà si realizzi nei tempi attesi da ciascuno.

Ma se tutto ciò non fosse possibile, ogni scelta avrebbe conseguenze indesiderate ed impreviste, $\mathrm{o}$ 'esternalità negative' che, potrebbero non produrre incertezza se esistesse qualcosa: politica o diritto naturale, in grado di orientare e di selezionare le preferenze individuali e di fornire indicazioni circa i tempi ed i costi della loro realizzazione. Implica l'esistenza di un qualcosa che selezioni le preferenze e i loro tempi di realizzazione.

Diversamente, l'incertezza si trasformerebbe in uno stato di disordine e poiché uno stato incerto non è sostenibile indefinitivamente, o perché almeno qualcuno desidera uscirne, esso si trasforma in maggiore richiesta di politica. Esiste una relazione tra la quantità di incertezza percepita e la disponibilità a spendere per avere certezza. Ma non essendo tale relazione razionale o fissa, bensì dipendente dalla percezione soggettiva dell'incertezza, la politica non può produrre razionalmente certezza. Da un altro e complementare punto di vista, la scarsità, o addirittura l'assenza di valori comuni, o di 'vincoli informali', è anche ciò che complica, o che può rendere impossibile, un calcolo economico-razionale per quanto riguarda la 'produzione di sicurezza' in relazione a minacce, o a comportamenti umani e ad eventi naturali ritenuti avversi. Non è un caso, quindi, che quando si possiedono scarsi elementi per valutare le intenzioni di un avversario, o si pensa che il suo comportamento sia ispirato da una logica di soprafazione o di annullamento, la propensione a spendere in sicurezza aumenti, e che i conflitti siano comportamenti caratterizzati da altissimi e distruttivi costi di transazione.

Tale impossibilità accresce, anche in maniera irrazionale, sia il bisogno di sicurezza ogni qual volta si avverta una minaccia (o se ne abbia sensazione), sia la richiesta, a questo punto anch'essa irrazionale, di politica; e induce quest'ultima a dilatare le proprie competenze nel vano tentativo di vincolare le scelte e le apprensioni individuali ad improbabili criteri di razionalità o di giustizia, a norme rigide (costituzione), o a fissarle essa 
stessa sperando così di ridurre la complessità delle scelte e le conseguenze prima menzionate.

5. Non è che la tradizione liberale ignorasse il problema del come si genera o si produce un ordine e di come mantenerlo, o non sapesse che senza un ordine, o la diffusa credenza di una tendenza ad esso, il clima sociale sarebbe diventato incerto e sarebbe degenerato in una forma di instabilità che presto o tardi avrebbe finito per proiettarsi negativamente sul funzionamento e sull'efficienza del mercato, e sulla sua stessa esistenza. Il fatto è che faceva teoria in un mondo in cui il mercato non si era ancora realizzato o non era ancora esteso come ora, e che pensava, o, detto diversamente, sperava (ed è, paradossalmente, la posizione dei suoi teorici più scettici) che il mercato, intendendo i prezzi come il più solerte ed efficiente sistema di trasmissione di conoscenza, avrebbe prodotto imitazione dei comportamenti 'virtuosi' e che ciò, unitamente al diffondersi di comportamenti complementari, avrebbe prodotto, sia pure come conseguenza non diretta, un ordine.

Aveva creduto di aver trovato una nuova teoria generale dell'azione umana che, trasformando il tempo in moneta, aveva posto le premesse per un calcolo razionale dei comportamenti e delle risorse umane incentrato sull'assunto della scarsità di tempo e di beni di contro all'illimitatezza dei beni e delle aspettative. Un assunto tutto sommato realistico che teneva conto del fatto che non tutto ciò che si desidera, o si auspica, può essere realizzato nei tempi e nei modi attesi da individui dotati di tempo scarso e di una conoscenza limitata e fallibile.

Tuttavia, quel che teorizzava la principale 'scuola' del liberalismo contemporaneo, quella 'Austriaca', non è stato falsificato, anche perché essa aveva piena consapevolezza che tale sua teoria sul ruolo svolto dalla diffusione e dalla trasmissione della conoscenza nel mercato avrebbe potuto dare i suoi frutti migliori in un ambiente istituzionale di tipo liberale e non di tipo democratico. In un ambiente istituzionale fatto di vincoli informali sufficientemente forti e di una limitazione costituzionale dell'entità ed ampiezza delle scelte pubbliche. E che avesse ragione lo si può constatare oggi, allorquando diventa palese che nonostante non 
esistano più limiti reali all'estensione delle scelte pubbliche, la maggior parte delle innovazioni che modificano la società non sono più prodotte da scelte politiche. Tant'è che oggi constatiamo che sono i 'fallimenti' della democrazia e della religione (ormai incapaci di produrre omogeneità sociale tramite l'educazione $o$ la pratica di valori religiosi ed etici), e non quelli del mercato, a costituire un ostacolo a quei comportamenti imitativi dai quali ci si aspettava tanto. Il mercato, detto altrimenti, riesce a soddisfare più aspettative di altre istituzioni e non è affatto detto che ne crei più di altre perché non può arrestarsi. A generare le aspettative, ormai, non sono tanto le istituzioni tradizionali, quanto lo sfrenato sviluppo della conoscenza e il continuo cambiamento delle posizioni iniziali che si lega al suo dinamismo.

Ciò detto, il mercato immaginato dai liberali classici era un qualcosa che avrebbe potuto dare i suoi frutti migliori in un ambiente di Rule of Law (che presuppone se non forti valori comuni, almeno una forte adesione a regole comuni). Ed essi - ed in particolare quella loro parte che, come i conservatori, aveva coltivato una salutare diffidenza nei riguardi della teoria democratica incautamente elevata a dogma- non ignoravano che ove invece avesse dovuto operare in un ambiente democratico, il mercato avrebbe finito per incrementare non i 'comportamenti imitativi virtuosi', ma semplicemente quelle aspettative individuali e sociali soggettive, instabili ed irrazionali, e quelle credenze che hanno soltanto una vaga relazione con la realtà, e che si legano a quella che, tutto sommato, resta l'illusoria speranza che da un controllo politico del processo di mercato sarebbe stato possibile trarre maggiori vantaggi di quanti se ne trarrebbero in un ambiente istituzionale di Rule of Law.

Soltanto ora ci si inizia a rendere conto che la già difficile convivenza tra democrazia e mercato — soprattutto quando si svolge in un contesto sociale caratterizzato dalla crisi dei valori religiosi, dall'inefficienza dei sistemi educativi e giudiziari, da una produzione e da un'allocazione legislativa dei diritti che non ha altro fine se non quello del consenso elettorale, e dalla trasformazione dei diritti naturali lockeani in diritti umani e sociali-moltiplica all'infinito le aspettative perchè lascia crescere la speranza che un controllo politico del processo di mercato 
possa tradursi in maggiori vantaggi per chi riesce a dirigerlo organizzando l'acquisizione del consenso intorno alle idee e alle aspettative maggiormente diffuse e condivise dall'opinione pubblica. Magari cavalcandole irresponsabilmente, salvo poi trovarsi nella condizione di non poterle, come ovvio, realizzare.

Che questi fossero pericoli latenti, mali endemici della teoria democratica - di un dio che, come scrive Hans-Hermann Hoppe, in Democracy. The God That Failed, del 2001, ha fallito- non era certo ignoto alla tradizione liberale. E, senza fare lunghe elencazioni, basti soltanto pensare a come Carl Menger, il padre della Scuola Austriaca, poneva la capacità di distinguere tra i bisogni a presupposto dell'affermazione di un ordine, o a come Bruno Leoni (che pure poneva lo 'scambio di pretese individuali reciproche' all'origine del diritto e quindi anche della convivenza politica) distingueva i regimi politici occidentali a seconda che fossero caratterizzati dal binomio legislazione-pianificazione o da quello Rule of Law e mercato, rilevando la difficoltà di convivenza tra mercato e legislazione.

Il modello di stato che si è affermato in Occidente in questi ultimi secoli, non è pertanto quello della tradizione costituzionale liberale, ma quello cosiddetto 'sociale', lucidamente previsto da Bastiat (che pure credeva ad una tendenziale armonizzazione degli 'interessi onesti') quando, parafrasandone la definizione, lo definiva come la "grande illusione caratterizzata dalla credenza che tutti possano prosperare a spese di tutti gli altri». Ma anche se la 'grande illusione' del liberalismo è stata, in definitiva, quella di pensare di poter sopravvivere in un mondo democratico perché la democrazia avrebbe ridotto le diseguaglianze tramite l'educazione di massa e prodotto valori comuni, se ciò non è avvenuto non si può certo dare la colpa al liberalismo e al mercato. Certamente, perdendo quella battaglia sul campo della comunicazione delle idee - che ha avuto la conseguenza di svuotare il termine liberalismo di connotati specifici e facilmente identificabili (una battaglia che si è persa nel momento in cui tutti si sono dichiarati 'liberali') — i teorici ed i seguaci del liberalismo hanno le loro responsabilità. Ma da questo a fare dell'individualismo liberale e del mercato concorrenziale gli unici responsabili della situazione attuale, il passo è lungo, e non tiene 
conto di molte cose che si è ormai in grado di valutare fuori dallo schema secondo il quale ogni cambiamento è per il meglio. Anche se non ce ne rendiamo conto.

Da questo punto di vista, quindi, nella nostra epoca la filosofia e la scienza politica hanno finora svolto essenzialmente una funzione di conservazione. E se è certamente vero che inventarsi una filosofia politica nuova, originale ed adeguata ai tempi e alla loro complessità non è impresa facile, ciò su cui bisogna riflettere è se i mutamenti ai quali si è accennato non sollecitino un ripensamento dei canoni ereditati dal passato più radicale di quello che realmente c'è stato, è anche vero che la scienza politica si è ristretta allo studio delle caratteristiche e alle dinamiche del 'modello stato' evitando, in quanto 'non scientifico', di immaginare qualcosa di diverso. In questo modo si sono entrambe rassegnate a dover riflettere sulle ripercussioni politiche di mutamenti esogeni alla politica e hanno perso molta di quell'aura di rispettabilità che derivava loro dall'essere in grado di elaborare criteri di distinzione sui comportamenti, sugli ideali, sulle ideologie e sulle istituzioni sociali e politiche.

I problemi del XXI secolo sono soltanto in parte quelli dei secoli precedenti e, a guardare la realtà con gli schemi concettuali che quei secoli hanno lasciato, si corre realmente il rischio di una path dependence priva di prospettive. 